\title{
RESEARCH
}

Open Access

\section{Circular RNAs are abundantly expressed and upregulated during repair of the damaged endometrium by Wharton's jelly-derived mesenchymal stem cells}

Baolan Sun ${ }^{1 \dagger}$, Lei Shi ${ }^{1+}$, Qin Shi ${ }^{1}$, Yao Jiang ${ }^{2}$, Zhangyao Su${ }^{3}$, Xiaoqing Yang ${ }^{1^{*}}$ and Yuquan Zhang ${ }^{1^{*}}$ (D)

\begin{abstract}
Background: Wharton's jelly-derived mesenchymal stem cells (WJ-MSCs) exhibit strong and powerful potential in repairing different diseases. The expression profile of circular RNA (circRNA) provides valuable insight for regulation of the repair process and exploration of reparative effect mechanisms.

Methods: Human endometrial stromal cells (ESCs) were cultured with mifepristone to obtain damaged ESCs, which were then cocultured with or without WJ-MSCs (cocultured group versus non-cocultured group) to observe the reparative effect upon damaged ESCs by WJ-MSCs. CircRNA microarray was performed between the two groups. Based on the transcriptomics data, the differential gene expression profiles of the two groups were analyzed by Gene Ontology (GO), Kyoto Encyclopedia of Genes and Genomes (KEGG) pathway, and network analysis methods. Screening of a circRNA database was performed, and the results were confirmed by quantitative polymerase chain reaction (qPCR).
\end{abstract}

Results: WJ-MSCs exerted a reparative effect upon damaged ESCs in the cocultured group such as improved cell morphology, higher proliferative ability, and lower apoptosis rate. CircRNA array showed that 7757 circRNAs were differentially expressed in ESCs from the cocultured group. Mitotic cell cycle, cell cycle process, and nuclear division ranked top in the GO upregulated list of the two groups, while DNA replication and cell cycle ranked top in the KEGG pathway analysis upregulated list of the two groups. The nine most aberrantly expressed circRNAs were selected for further verification in the same cohort of samples by microarray analysis. Seven of the nine most aberrantly circRNAs were confirmed to be significantly upregulated in the cocultured group. And four of the seven circRNAs (hsa_circ_0015825, hsa-circRNA4049-38, hsa-circRNA5028-15, and hsa_circ_0111659) expression both in ESCs and WJ-MSCs tended to decrease with time by qPCR. The levels of the remaining three circRNAs (hsacircRNA8881-21, hsa_circ_0020492 and hsa_circ_ 0026141) did not change significantly over time in either ESCs or WJ-MSCs. Moreover, we focused on hsa_circRNA_0111659 and predicted its miRNAs and targeted mRNA. The association of circRNA-miRNA-mRNA is likely to be involved in regulating the repair of endometrial damage.

Conclusions: Our results presented the abundant and upregulated circRNAs profile during repair of the damaged endometrium by WJ-MSCs and provided a novel perspective for circRNAs in the regulation of WJ-MSCs for endometrial repair.

Keywords: Circular RNA, Endometrial stromal cells, Wharton's jelly-derived mesenchymal stem cells, Repair

\footnotetext{
*Correspondence: ntyxq169@126.com; jsnt_zhangyuquan@163.com

${ }^{+}$Baolan Sun and Lei Shi contributed equally to this work.

'Department of Obstetrics and Gynecology, Affiliated Hospital of Nantong

University, Nantong, People's Republic of China

Full list of author information is available at the end of the article
}

(c) The Author(s). 2018 Open Access This article is distributed under the terms of the Creative Commons Attribution 4.0 International License (http://creativecommons.org/licenses/by/4.0/), which permits unrestricted use, distribution, and reproduction in any medium, provided you give appropriate credit to the original author(s) and the source, provide a link to the Creative Commons license, and indicate if changes were made. The Creative Commons Public Domain Dedication waiver (http://creativecommons.org/publicdomain/zero/1.0/) applies to the data made available in this article, unless otherwise stated. 


\section{Background}

The human endometrium not only maintains and affects normal menstruation but also plays a vital role in female fertility $[1,2]$. Menopause or amenorrhea due to endometrial damage is one of the most important factors influencing infertility. Previous studies have found that the pathological mechanism underlying endometrial damage involves damage to the basal layer of the endometrium; this changes the regular growth and abscission of the endometrium during the normal menstrual cycle [3, 4]. The main route of endometrial repair after injury involves the repair or production of new basal layer cells via endogenous or exogenous pathways [5].

Human umbilical cord mesenchymal stem cells (WJMSCs), which exist in the umbilical cord Wharton's jelly and the perivascular area, are increasingly being used in the field of regenerative medicine [6]. Studies have shown that WJ-MSCs exhibit a strong and powerful ability to repair different diseases, including cardiovascular disease, hematopathy, diabetes, muscular degeneration, liver disease, and endometrial damage [7-13]. Our previous studies showed that WJ-MSCs are able to ameliorate damaged human endometrial stromal cells (ESCs) both in vitro and in vivo $[13,14]$, thus indicating that it is the possible to use WJ-MSCs to repair endometrial damage. However, in vivo, we also found that a large number of WJ-MSCs $\left(10^{6}-10^{7}\right)$ are required to achieve a repair effect. Because the acquisition, cultivation, and storage of WJ-MSCs require a substantial amount of manpower and material resources, it is imperative to improve the efficiency of working with WJ-MSCs. For example, it is important to investigate the regulatory mechanisms involved in the repair of ESCs by WJ-MSCs.

Circular RNA (circRNA) is a unique class of endogenous non-coding RNA (ncRNA) forming a closed continuous loop by back-splicing with covalently joined $3{ }^{\prime}$ - and $5^{\prime}$-ends. Because of their closed structure, circRNAs are very stable and are resistant to RNA degradation [15-17]. Most often, they are derived from annotated host gene exons, which may give rise to alternative circRNAs, depending on the exact exons involved in back-splicing; they also compose a large class of animal RNAs with developmental and tissue-specific expression patterns [18]. Recent studies have revealed that circRNAs regulate gene expression via a range of different mechanisms, specifically, by serving as miRNA sponges $[19,20]$, facilitating transcription of their host gene by directly associating with RNA polymerase II [21]. Furthermore, circRNAs are extensively involved in the pathological process of damage and repair induced by different causes, such as the priming phase of rat liver regeneration, neuronal injury or damage caused by chemical carcinogens [22-25]. However, whether circRNAs are involved in the process of WJ-MSC-mediated endometrial repair remains unclear, as do the specific mechanisms involved.
In this study, we aimed to explore the underlying role of circRNAs in the WJ-MSC-mediated repair of damaged ESCs and focused particularly on circRNAs vital for the repair of damaged ESCs. To do so, we used circRNA microarray to detect differentially expressed circRNA profiles in damaged ESCs cocultured with or without WJ-MSCs.

\section{Methods \\ Cells and culture}

To obtain WJ-MSCs, we collected human umbilical cords from healthy full-term deliveries. We also obtained endometrium samples from patients with intramural or pedunculated sub-serosal hysteromyoma without endometrial abnormalities to obtain ESCs. None of the women had taken medication or had received hormonal therapy for at least 6 months before undergoing hysterectomy. All samples were obtained after the patients provided signed informed consent, and the procedures were approved by the Ethics Committee of the Affiliated Hospital of Nantong University. Both WJ-MSCs and ESCs were isolated, cultured, and identified as previously described [26]. WJ-MSCs and ESCs were cultured in Dulbecco's modified Eagle's medium F12 (DMEM/F-12; Hyclone, Logan, UT, USA) containing 10\% fetal bovine serum (FBS; Gibco, Waltham, MA, USA), $100 \mathrm{U} / \mathrm{ml}$ penicillin, and $100 \mu \mathrm{g} / \mathrm{ml}$ streptomycin at $37^{\circ} \mathrm{C}$ in a humidified $5 \% \mathrm{CO}_{2}$ atmosphere, respectively.

ESCs treated with mifepristone and cocultured with WJ-MSCs Establishment of the damaged-ESC model and the WJ-MSCs coculture system was performed according to our previous protocol [13]. In brief, the ESCs of passage 2 were dispensed into 24-well plates at a density of $1.5 \times$ $10^{4}$ cells/well and cultured in DMEM-F12 with $10 \%$ FBS. After $24 \mathrm{~h}$, this medium was changed to a medium containing $2 \%$ FBS for $12 \mathrm{~h}$. Then, the cells were treated with mifepristone (M8046; Sigma-Aldrich, Saint Louis, MO, USA) at a concentration of $60 \mu \mathrm{mol} / \mathrm{L}$. After $48 \mathrm{~h}$, the medium was replaced by fresh medium ( $2 \% \mathrm{FBS})$ to allow culture to proceed, but without mifepristone. The Transwell system $(24 \mathrm{~mm}$ Transwell with a $0.4-\mu \mathrm{m}$ pore polycarbonate membrane insert, \#3412; Corning) was used to establish the coculture system. WJ-MSCs from passage 3 were seeded on top of the artificial basement membrane and placed in the upper part of the tissue culture plate for coculture with damaged ESCs for $24 \mathrm{~h}$, $48 \mathrm{~h}$, and $72 \mathrm{~h}$. Control plates were not seeded with WJ-MSCs. ESCs and WJ-MSCs were subsequently digested and collected for use. We took the damaged ESCs and cocultured these with WJ-MSCs as the cocultured group; damaged ESCs without coculture with WJ-MSCs were used as the non-cocultured group. 


\section{Morphological changes of ESCs}

The Transwell chambers in the culture plates were removed, leaving the lower layer of ESCs. The morphological changes of ESCs in each group were then observed by optical microscopy (Olympus, Japan), and the morphological effects of WJ-MSCs on the repair of damaged ESCs were investigated.

\section{CCK-8 assay as an indicator of ESC proliferation}

The proliferation of ESCs was evaluated using a Cell Counting Kit (CCK-8; Beyotime, Jiangsu, China) according to the manufacturer's instructions. After cocultured with WJ-MSCs for $24 \mathrm{~h}, 48 \mathrm{~h}$, and $72 \mathrm{~h}$, the proliferative ability of damaged ESCs in the cocultured or non-cocultured groups was determined. At the end of each experiment, $10 \mu \mathrm{l} /$ well of CCK-8 assay solution was added to each culture, and samples were incubated for $2 \mathrm{~h}$. Absorbance was recorded at $450 \mathrm{~nm}$ on an automatic microplate reader (Bio Teck, CA, USA) within $30 \mathrm{~min}$. Cells in each group were prepared in triplicate.

\section{Detection of apoptosis in ESCs by flow cytometry}

To detect apoptosis, the ESCs from each group were collected and washed twice with cold PBS and then resuspended in $1 \times$ binding buffer at a concentration of $1 \times 10^{6} / \mathrm{ml}$. A $100 \mu \mathrm{l}$ volume of this solution was then transferred to a $5-\mathrm{ml}$ culture tube, and $5 \mu \mathrm{l}$ fluorescein isothiocyanate-Annexin $\mathrm{V}$ and $5 \mu \mathrm{l}$ propidium iodide (Becton Dickinson, San Jose, CA, USA) were added. After incubation for $15 \mathrm{~min}$ at $25^{\circ} \mathrm{C}$ in the dark, $400 \mu \mathrm{l}$ of $1 \times$ binding buffer was added to each tube. Stained cells were then sorted with a FACScan flow cytometer (Becton Dickinson, NJ, USA) within $1 \mathrm{~h}$. Cells in each group were prepared in triplicate.

\section{Circular RNA microarray analysis}

A CapitalBio Technology Human CircRNA Array v2 was designed with four identical arrays per slide $(4 \times 180 \mathrm{~K}$ format), with each array containing probes interrogating approximately 170,340 human circRNAs. The circRNA array also contained 4974 Agilent control probes. In total, 7755 circRNAs were detected. All microarray analysis was performed by CapitalBio Technology (Beijing, China). The circRNA array data were analyzed for data summarization, normalization, and quality control with GeneSpring software V13.0 (Agilent). To select differentially expressed genes, we used threshold values of $\geq 2$ and $\leq-2$-fold change, and a $t$ test $P$ value of 0.05 . Data were $\log _{2}$ transformed and median-centered by genes in CLUSTER 3.0 software, then further analyzed by hierarchical clustering with average linkage. Finally, tree visualization was performed in Java Treeview (Stanford University School of Medicine, Stanford, CA, USA).

\section{Extraction of total RNA and quantitative polymerase} chain reaction (qPCR)

Total RNA was extracted from all groups by using Trizol reagent (Invitrogen, Carlsbad, CA, USA) and purified with a mirVana miRNA Isolation Kit (Ambion, Austen, TX, USA) according to the manufacturer's protocol. The purity and concentration of RNA were then determined from OD260/280 readings with a NanoDrop 2000 spectrophotometer (Thermo Scientific). RNA integrity was then determined by $1 \%$ formaldehyde denaturing gel electrophoresis. According to the manufacturer's instructions, we used a PrimeScript RT reagent kit (Takara Bio, Japan) to perform reverse transcription with the quantified RNA to synthesize complementary DNA (cDNA). QPCR was performed using SYBR-Green Premix Ex Taq (Takara Bio, Japan) and evaluated using an ABI PRISM 7500 Sequence Detection System. GAPDH was used to normalize RNA preparations. The relative expression levels of selected circRNAs and host gene mRNA were determined using the $2^{-\triangle \Delta C T}$ method. Primer sequences for circRNAs and host genes for qPCR are listed in Table 1 and Table 2, respectively.

\section{Bioinformatics analysis}

CircRNA can target miRNA and indirectly regulate mRNA translation. To further elucidate the role of circRNAs in the repair of endometrial damage, we first selected differentially expressed circRNAs and then predicted the target miRNAs for each selected circRNA in miRanda software. According to the relationship between circRNAs and target miRNAs, the first ten circRNAs with the largest fold change (FC) were selected to create a circRNA-miRNA network diagram. circRNA function was predicted by Gene Ontology (GO) functional annotation of co-expressed genes. Gene functions were classified into three subgroups: biological process, cellular component, and molecular function. GO terms with a $P$ value less than 0.05 were considered statistically significant. The top 30 enriched $\mathrm{GO}$ terms among the two groups, ranked by fold enrichment and enrichment score, are presented herein. KEGG pathway analysis was then performed to determine the involvement of co-expressed genes in different biological pathways.

Because we previously found that the expression of VEGF is upregulated and involved in the WJ-MSC-mediated repair of damaged ESCs [13], the prediction of circRNA-miRNA-VEGF gene associations was also determined by using two databases: Targetscan (http:// www.targetscan.org/vert_72/) and miRwalk (http://zmf.umm. uni-heidelberg.de/apps/zmf/mirwalk2/index.html).

\section{Statistical analysis}

Data were analyzed in SPSS20.0 software. All quantitative data are expressed as mean \pm standard deviation (SD). Student's $t$ test was used to compare data between the cocultured and non-cocultured groups. One-way 
Table 1 The characteristics and primers of the nine circRNAs

\begin{tabular}{|c|c|c|c|c|c|}
\hline Serial number & circRNAs & gene & Sequence $\left(5^{\prime}->3^{\prime}\right)$ (Forward/Reverse) & Template strand & Product length \\
\hline \multirow[t]{2}{*}{$\overline{1}$} & hsa-circRNA8881-21 & ASPM & TGTCTCTTCTGTAAAGATGCCGAA & Plus & 92 \\
\hline & & & ACCAATTCGAAGCCACAAAGGA & Minus & \\
\hline \multirow[t]{2}{*}{2} & hsa_circ_0020488 & MKI67 & CAGTGACCAGCCACAGGAGA & Plus & 188 \\
\hline & & & CGACCCCGCTCCTTTTGATA & Minus & \\
\hline \multirow[t]{2}{*}{3} & hsa_circ_0020492 & MKI67 & AATCCATGAGCAGGAGGCAAT & Plus & 166 \\
\hline & & & GGGGAAGGCCAGAAGCAAA & Minus & \\
\hline \multirow[t]{2}{*}{4} & hsa_circ_0026141 & TROAP & TAACCGCCATCCACTGCTTC & Plus & 178 \\
\hline & & & GGGCGAGTGGAAGGGTGAAA & Minus & \\
\hline \multirow[t]{2}{*}{5} & hsa-circRNA4049-38 & WDR62 & GTTCCTCCGCCACCACTTTGA & Plus & 195 \\
\hline & & & TCATGGGGGTAAAGTAGCAATCCA & Minus & \\
\hline \multirow[t]{2}{*}{6} & hsa_circ_0015825 & KIF14 & AGGGGTGAAGATGCCTITGTG & Plus & 164 \\
\hline & & & GACCCTAAGCTCTTCTTTGGACAT & Minus & \\
\hline \multirow[t]{2}{*}{7} & hsa-circRNA5028-15 & MYBL2 & GAAAGTCCGGAAGTCTCTGGC & Plus & 171 \\
\hline & & & GTGGTTGTGCCAGCGTTCA & Minus & \\
\hline \multirow[t]{2}{*}{8} & hsa_circ_0020487 & MKI67 & CTCTATCCCAGTGACCAGCCA & Plus & 145 \\
\hline & & & ATTGCCTCCTGCTCATGGATTT & Minus & \\
\hline \multirow[t]{2}{*}{9} & hsa_circ_0111659 & KIF14 & GGCTGAGTGATTTACTGCCTTGT & Plus & 174 \\
\hline & & & GGTCTTITCCTGCAGAGGTGTT & Minus & \\
\hline \multirow[t]{2}{*}{10} & & GAPDH & GGACTCATGGTATGAGAGCTGG & Plus & 180 \\
\hline & & & CAGCGTACTCCCCACATCAC & Minus & \\
\hline
\end{tabular}

analysis of variance was used to analyze the circRNAs levels of ESCs or WJ-MSCs after coculture for $24 \mathrm{~h}$, 48 h, and 72 h. $P<0.05$ was considered to indicate a statistically significant difference.

\section{Results}

WJ-MSCs exert a reparative effect upon damaged ESCs After the treatment of ESCs with mifepristone for $48 \mathrm{~h}$, optical microscopy revealed disordered distribution, larger cell intervals, vacuolization phenomena, and poor shading of damaged cells compared with normal ESCs. After the damaged ESCs were cocultured with WJ-MSCs for $48 \mathrm{~h}$, the growth state of cells was similar to that of normal cells, and the number of cells was significantly higher, the arrangement of cells was more regular and dense, although the density was still lower than that in the normal group. However, although the cell morphology

Table 2 The primers of the host genes

\begin{tabular}{|c|c|c|c|c|}
\hline Serial number & gene & $\begin{array}{l}\text { Sequence }\left(5^{\prime}->3^{\prime}\right) \\
\text { (Forward/Reverse) }\end{array}$ & Template strand & Length \\
\hline \multirow[t]{2}{*}{1} & \multirow[t]{2}{*}{ ASPM } & GAGCATTTCTGTCTGCAAAACATC & Plus & 24 \\
\hline & & TTGCAGGCAGCTTTCACTTTAC & Minus & 22 \\
\hline \multirow[t]{2}{*}{2} & \multirow[t]{2}{*}{ MKI67 } & TTGTAAATTTGCTTCTGGCCTTC & Plus & 23 \\
\hline & & ACGGATGTCACATTCAATACCC & Minus & 22 \\
\hline \multirow[t]{2}{*}{3} & \multirow[t]{2}{*}{ TROAP } & GGATCAGTCAGCCTCGGAAC & Plus & 20 \\
\hline & & CAGGGTCAGCCACAAACTCT & Minus & 20 \\
\hline \multirow[t]{2}{*}{4} & \multirow[t]{2}{*}{ WDR62 } & TGAGAAGAGGGTGCTGGAGA & Plus & 20 \\
\hline & & CCTGTGCCACGTCTACCC & Minus & 18 \\
\hline \multirow[t]{2}{*}{5} & \multirow[t]{2}{*}{ KIF14 } & GACTTCAGGGCCTCTCGG & Plus & 18 \\
\hline & & GACCCTAAGCTCTTCTITGGAC & Minus & 22 \\
\hline \multirow[t]{2}{*}{6} & \multirow[t]{2}{*}{ MYBL2 } & GAGGGATAGCAAGTGCAAGGT & Plus & 21 \\
\hline & & TTCCAGTCCTGCTGTCCAAA & Minus & 20 \\
\hline
\end{tabular}


of ESCs in the non-cocultured group recovered to a certain extent, and the number of nuclear vacuoles was lower than that initially seen in the damaged cells, the cells were still rare and disordered, as compared with cells in the normal group (Fig. 1a).

After coculture for $48 \mathrm{~h}$, the proliferative ability of cells in the cocultured group was higher than that in the non-cocultured group, and this difference continued up to $72 \mathrm{~h}(P<0.05)$. The proliferative ability of damaged ESCs in the non-cocultured group was significantly lower than that in the normal group after $24 \mathrm{~h}, 48 \mathrm{~h}$, and $72 \mathrm{~h}(P<0.01$; Fig. $1 \mathrm{~b})$.

The apoptosis rate of the cocultured group (36.29 \pm $3.70 \%)$ was significantly lower than that of the non-cocultured group $(68.58 \pm 5.47 \%, P<0.01)$ but was still higher than that of ESCs in the normal group $(17.72 \pm 7.42 \%, P<0.05)$. However, the apoptosis rate of ESCs in the non-cocultured was significantly higher than that of the normal group $(P<0.01$; Fig. 1c, d).

\section{Analysis of circRNA expression profiles}

CircRNA expression profiles are presented in the form of a heat map (Fig. 2a). The differences in circRNA expression profiles between the two groups are further shown as a scatter plot (Fig. 2b) and volcano plot (Fig. 2c). Overall, 7757 circRNAs were significantly differentially expressed (fold change $>2.0$ or $<-2.0, P<0.05$ ). In comparison with samples from the non-cocultured group, 5423 circRNAs were upregulated (Additional file 1: Table S1) and 2334 circRNAs (Additional file 1: Table S2) were downregulated in samples from the cocultured group (Fig. 2b).

Subsequently, we narrowed the scope of our analysis to the nine most aberrantly expressed circRNAs. Table 1 shows the nine circRNAs that showed the largest FC in our microarray. We found that all nine of these circRNAs were upregulated, according to our microarray analysis. We further confirmed the expression levels of these nine circRNAs by qPCR using the same cohort of samples in RNA microarray analysis. Seven of the nine circRNAs were confirmed to be

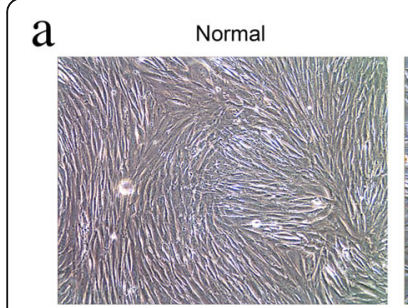

damaged and coculture

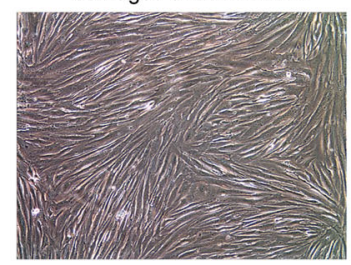

b

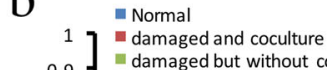

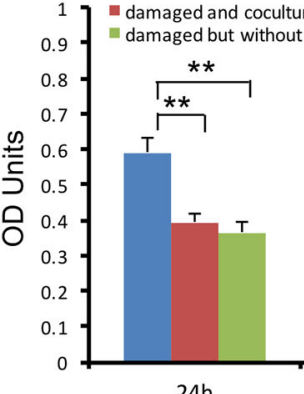

$24 \mathrm{~h}$
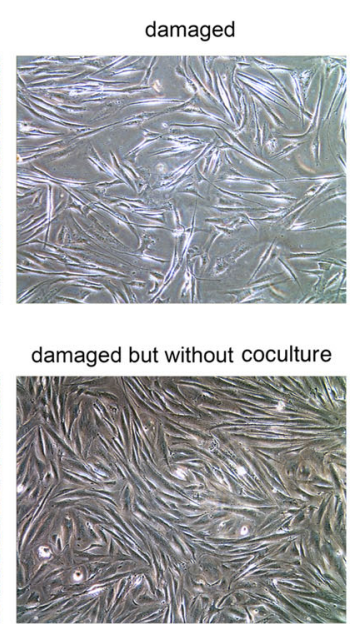

damaged but without coculture

are $* \star$
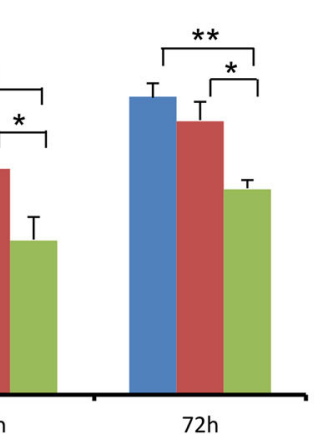

c
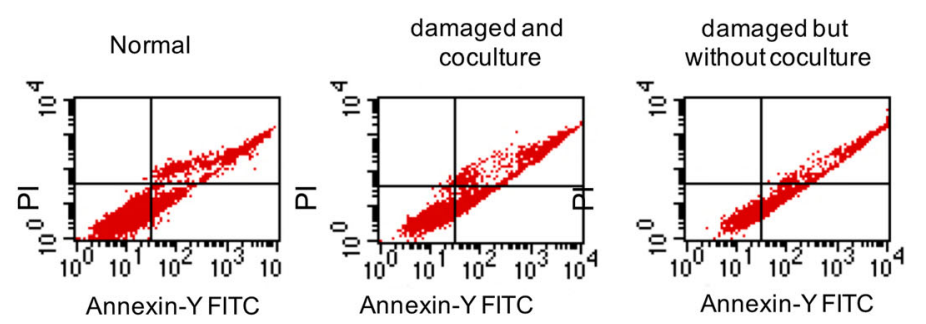

d

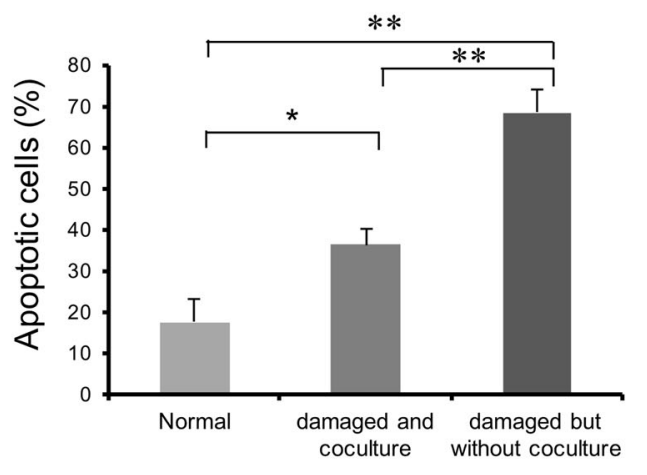

Fig. 1 WJ-MSCs had a reparative repair effect on damaged ESCs. a Morphological changes of ESCs after treatment with mifepristone (60 $\mu$ mol/L) for 48 h. b Absorbance of ESCs in different groups at 24 h, 48 h, and 72 h, as shown by CCK-8 assays. c, d The proportions of apoptotic cells in the three groups were evaluated by Annexin V/propidium iodide staining and flow cytometry. The proportion of apoptotic cells was higher in the non-cocultured group than the cocultured group and normal group. Data are expressed as mean \pm standard deviation (SD). Scale bars $=10 \mu \mathrm{m} .{ }^{*} P<0.05 ;{ }^{*} P<0.01$ 


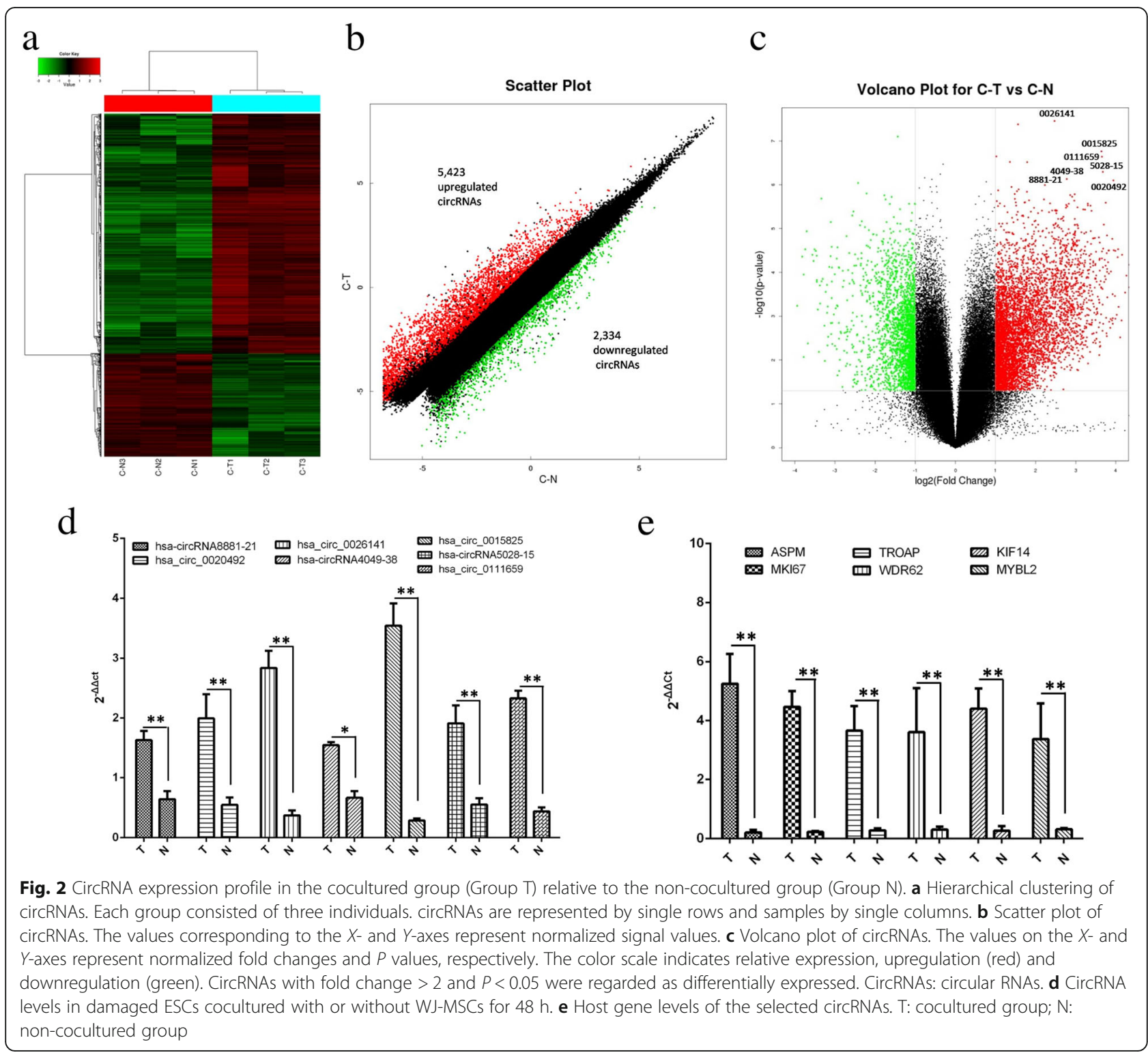

significantly upregulated in the cocultured group $(P<0.05)$, including hsa-circRNA8881-21, hsa_circ_0020492, hsa_circ_ 0026141, hsa-circRNA4049-38, hsa_circ_0015825, hsa-circRNA5028-15 and hsa_circ_ 0111659 (Fig. 2c, d), whose expression levels were consistent with the RNA microarray analysis. However, the expression levels of hsa_circ_0020487 and hsa_circ_0020488 were too low to be detected. Meanwhile, we also evaluated host genes of the seven validated circRNAs (Table 2), including abnormal spindle-like microcephaly (ASPM), marker of proliferation Ki-67, (MKI67), trophinin-associated protein (TROAP, also named TASTIN), WD repeat domain 62 (WDR62), kinesin family protein 14 (KIF14), and V-Myb avian myeloblastosis viral oncogene homolog-like 2 (MYBL2) using qPCR. Compared with those in the non-cocultured samples, the expression levels of the host genes in the cocultured group were significantly higher, although the expression level of host genes was not completely consistent with that of their respective circRNAs (Fig. 2e).

Furthermore, we observed trends in the dynamic expression trend of seven circRNAs in both ESCs and WJ-MSCs of the cocultured group during the repair process. The expression levels of the seven circRNAs at $24 \mathrm{~h}, 48 \mathrm{~h}$, and $72 \mathrm{~h}$ were compared using qPCR. Trend comparison showed that the expression of the four circRNAs continued to decrease over time while the expression of four circRNAs (hsa-circRNA4049-38, hsa_circ_0015825, hsa-circRNA5028-15, and hsa_circ_0111659) in ESCs at $24 \mathrm{~h}$ were significantly higher than at $48 \mathrm{~h}$ or $72 \mathrm{~h}(P<0.01$ or $P<0.05$ ). However, the levels of the remaining three circRNAs (hsa-circRNA8881-21, hsa_circ_0020492, and hsa_circ_0026141) did not change significantly over 
time (Fig. 3). Meanwhile, we evaluated the expression profiles of seven circRNAs in WJ-MSCs from the cocultured group. The expression profiles of the seven circRNAs in WJ-MSCs were similar to that seen in ESCs (Fig. 4).

\section{Enrichment analysis of the biological function of circRNA co-expressed genes}

The GO analysis results between cocultured group and non-cocultured group showed that the upregulated circRNAs were significantly enriched in the BP, including mitotic cell cycle, cell cycle process, and nuclear division (Additional file 1: Table S3). The CC analysis showed that the upregulated circRNAs were significantly enriched in the chromosome, intracellular non-membrane-bounded and nuclear replication fork (Additional file 1: Table S4). In addition, in the MF analysis, the upregulated circRNAs were enriched in ATP binding and DNA-dependent ATPase activity (Additional file 1: Table S5). We generated a list of the circRNAs with the top $10 P$ values according to the GOTERM_BP, CC, MF_FAT list for biological processes, cellular component, and molecular function of general interest. After comparing the BP, CC, and MF, it was found that the enrichment of circRNAs related to the repair in the cocultured group was upregulated. The upregulated circRNA enrichment in cocultured group occurred in the mitotic cell cycle, cell cycle process, nuclear division, chromosome, ATP binding, and adenyl ribonucleotide binding (Fig. 5a-c). The most significantly enriched pathways of the upregulated circRNAs in the
KEGG analysis conducted on DAVID are shown in Fig. 5d. Between cocultured group and non-cocultured group, the upregulated circRNAs were enriched in DNA replication, cell cycle, Fanconi anemia pathway, homologous recombination, aminoacyl-tRNA biosynthesis, and nucleotide excision repair. Specific information on the top ten pathways in the KEGG pathway analysis for upregulated circRNAs is listed in Additional file 1: Table S6.

\section{Prediction of circRNA-miRNA-mRNA}

CircRNA can target miRNA and indirectly regulate the translation of mRNA. MiRanda software was used to select the circRNAs showing differential expression and thus predict the target miRNAs; a list of the miRNAs generated is given in Additional file 1: Table S7. According to the relationship between circRNAs and target miRNAs, the first ten circRNAs with the largest FC were selected to create a circRNA-miRNA network map. Only one of the ten circRNAs did not predict a target miRNA. The remaining nine circRNAs were predicted to 545 target miRNAs. A network map was constructed containing 9 circRNAs and 545 miRNAs (Fig. 6). To investigate whether VEGF was regulated by ncRNAs, we also predicted the target miRNAs of VEGF by using the miRwalk database and identified 102 targeted VEGF miRNAs (Additional file 1: Table S8). Of these, miR-17-5p, miR-20b-5p, and miR-93-5p were predicted to be common targets of hsa_circRNA_0111659. The co-expression network showed that miR-17-5p, miR-20b-5p, and miR-93-5p were co-related with hsa_circRNA_0111659 (Fig. 7a). Using

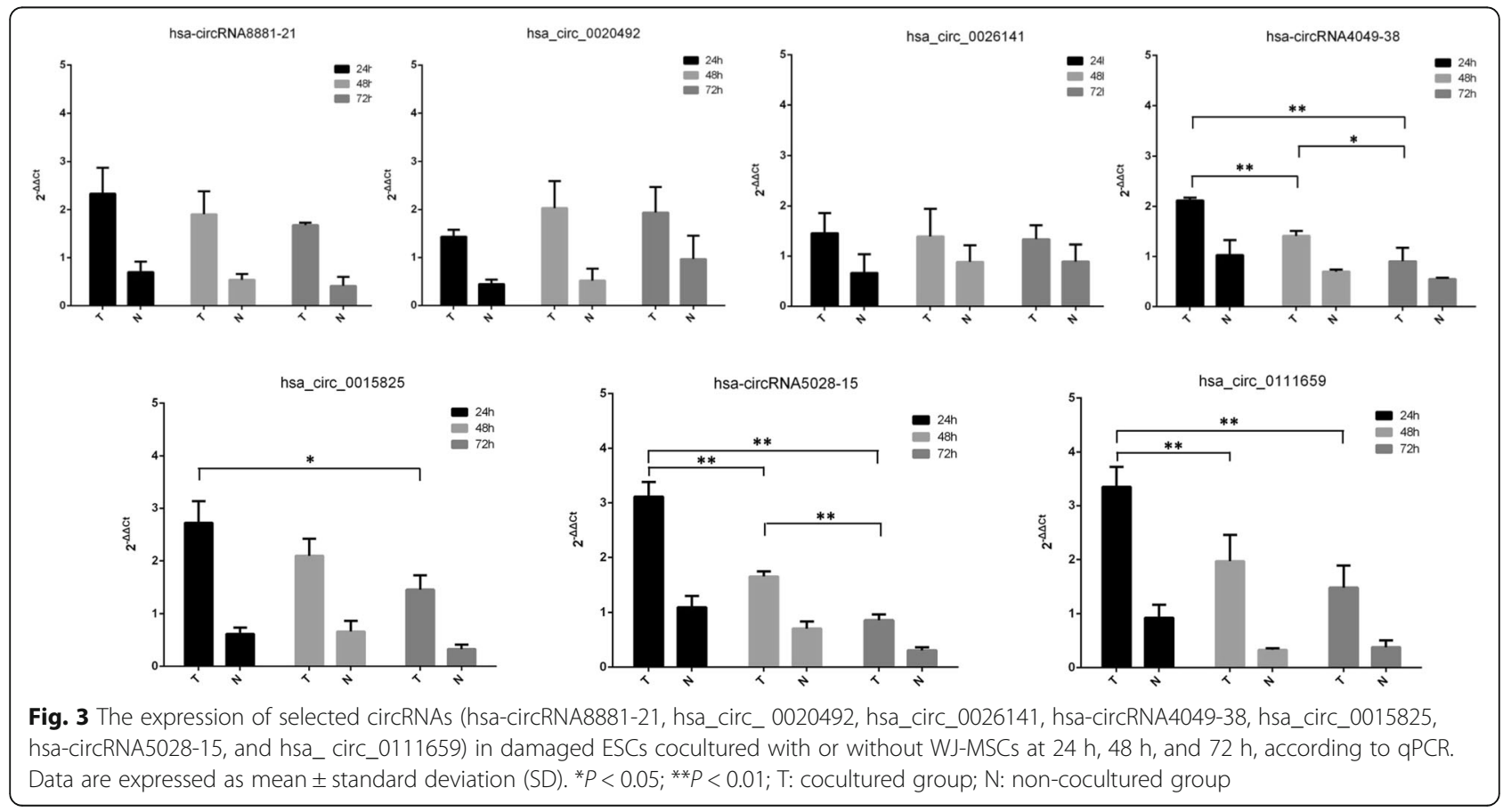




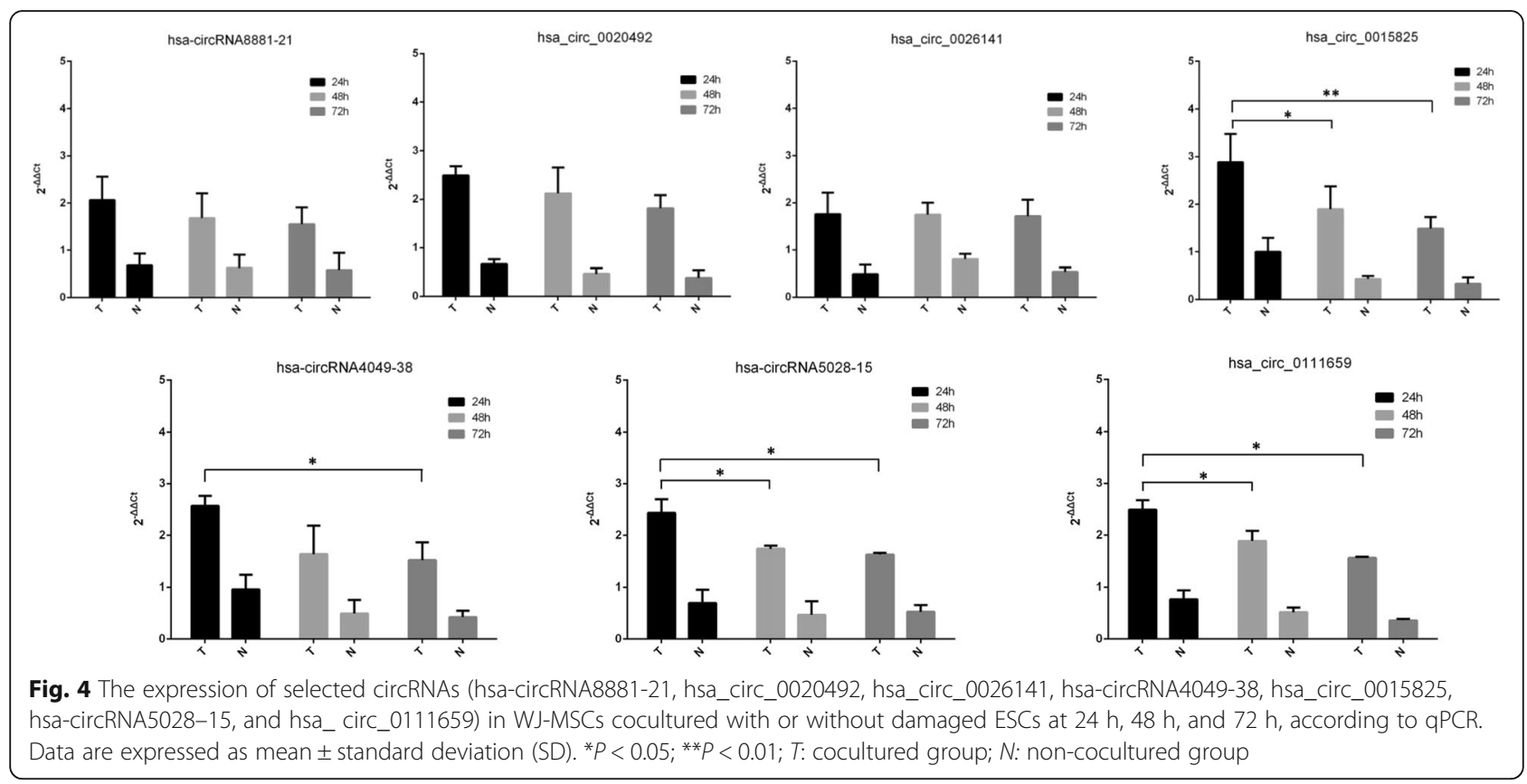

circBase to further predict miRNAs associated with circRNA_0111659 binding, we found that miR-17-5p, miR-20b-5p, and miR-93-5p have multiple binding sites with has_circRNA_0111659 (Fig. 7b1-b3). Further details of the molecular interactions between VEGF, hsacircRNA_0111659 and miR-17-5p, miR-20b-5p, and miR-93-5p are depicted in Fig. 7.

\section{Discussion}

WJ-MSCs are believed to facilitate the repair of damaged tissue and organs by differentiating into specific cells or/ and by secreting a large number of bioactive factors such as VEGF, hepatocyte growth factor, hepatocyte growth factor, and interstitial cell-derived factor-1 (stromal cell). Previous studies and the current study clearly demonstrate that WJ-MSCs exert a reparative effect on damaged ESCs $[13,14]$. In the present study, we first found that circRNAs are abundantly expressed and upregulated during the repair of damaged endometrium by WJ-MSCs; this may play an important role in improving the effect of WJ-MSCs on the repair of endometrial damage.

Recent studies have also shown that circRNAs are involved in the process of tissue damage repair. Li and colleagues [22] have used high-throughput sequencing technology to identify six key circRNAs showing significant changes during the repair of liver injury. In another study, Lin et al. [23] have also

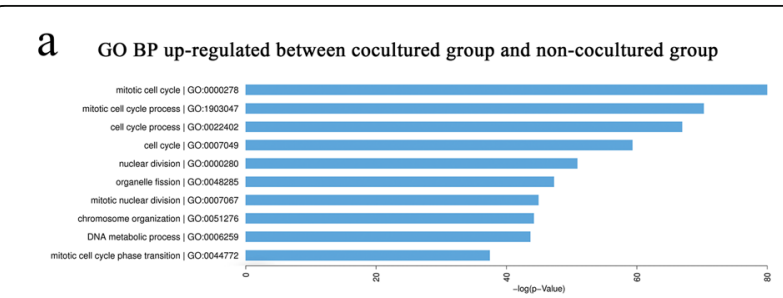

C GO MF up-regulated between cocultured group and non-cocultured group

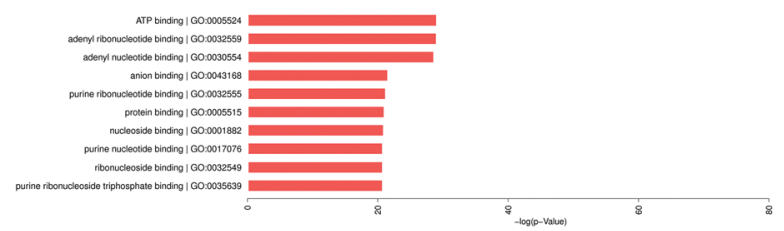

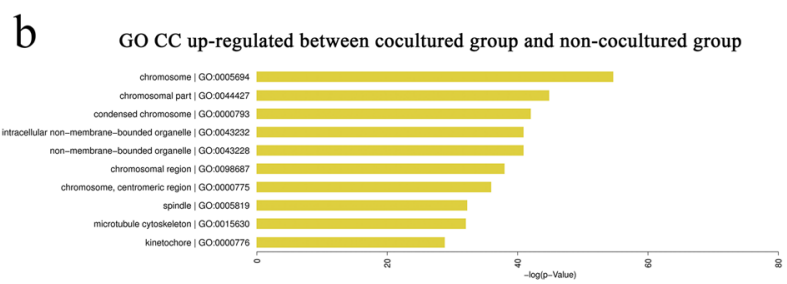

d

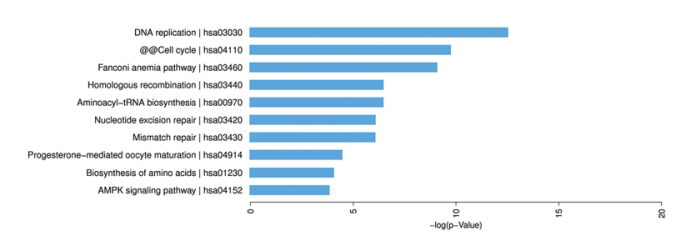

Fig. 5 GO and KEGG analysis for circRNA-targeted mRNAs. GO enrichment for the target mRNAs, including biological processes (a), cell component (b), and molecular function (c). d KEGG pathway analysis. GO: Gene Ontology; KEGG: Kyoto Encyclopedia of Genes and Genomes; circRNAs: circular RNAs 


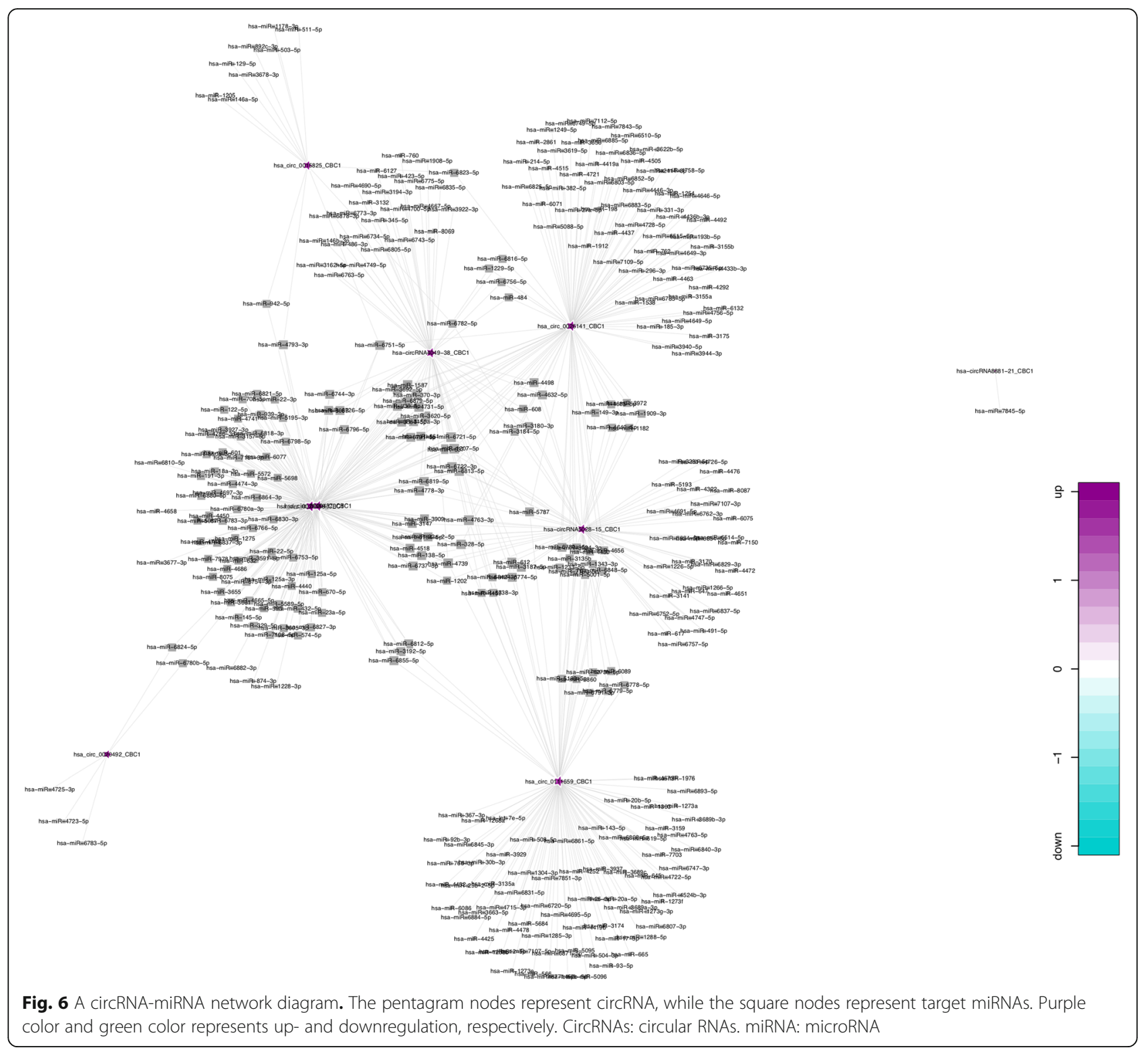

reported the upregulation of the expression three circRNAs and the downregulation of 12 circRNAs in the HT22 cell model of cerebral ischemia-reperfusion injury. Collectively, these results suggest that circRNAs are widely involved in the pathological process of damage repair and exert regulatory function at the transcriptional or post transcriptional level. circRNA expression profiles were found to be tissue-specific, thus enabling prediction of potential biological functions. However, whether circRNAs play a role in the repair of endometrial injury remains unclear.

In the present study, we found that circRNAs are abundantly expressed and upregulated during the repair of ESCs by WJ-MSCs. More than 140,000 circRNAs were detected in our study. Of these, approximately 5.5\% ( 7757 circRNAs) showed dysregulated expression in the damaged ESCs that were cocultured with WJ-MSCs for $48 \mathrm{~h}$. Specifically, 5423 circRNAs were upregulated, and 2334 circRNAs were downregulated. We then focused on nine circRNAs in the cocultured group showing the largest FC in expression. All were shown to be upregulated by microarray, and seven of these nine circRNAs were significantly upregulated in microarray analysis and other cohort samples, including ESCs and WJ-MSCs. These results were not surprising, because circRNAs are known to be upregulated during cell cycle regulation and DNA damage repair [24, 25]. However, we found that the expression of four circRNAs gradually decreased in both ESCs and WJ-MSCs over time in vitro experiment. These observations can be explained, in part, by circRNAs being passively diluted during cell 


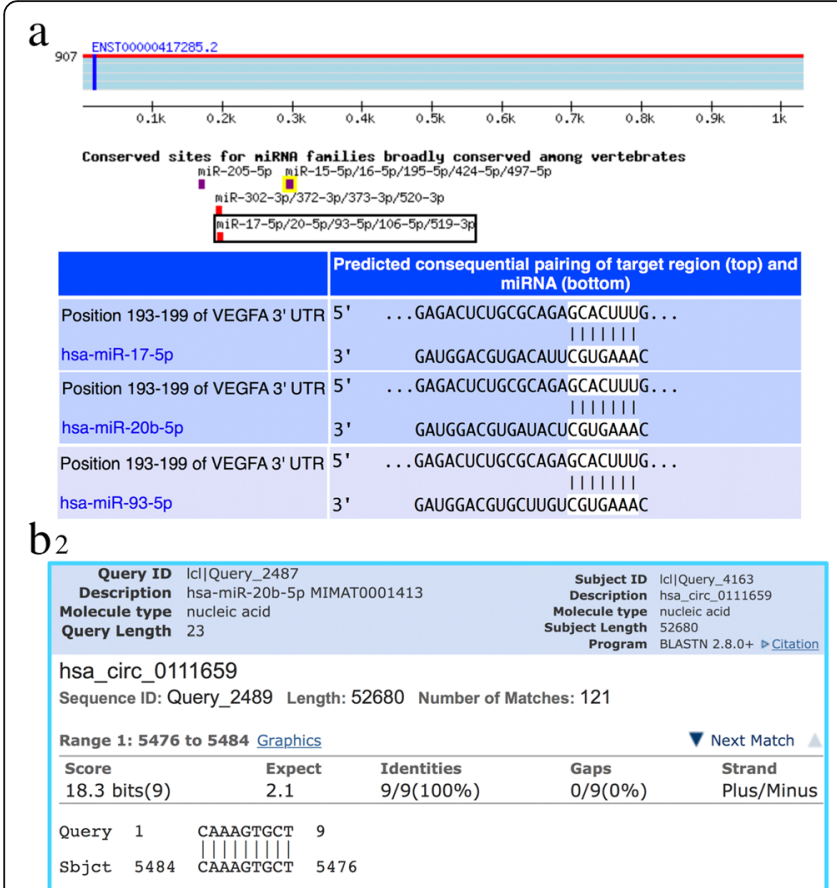

$\mathrm{b}_{1}$

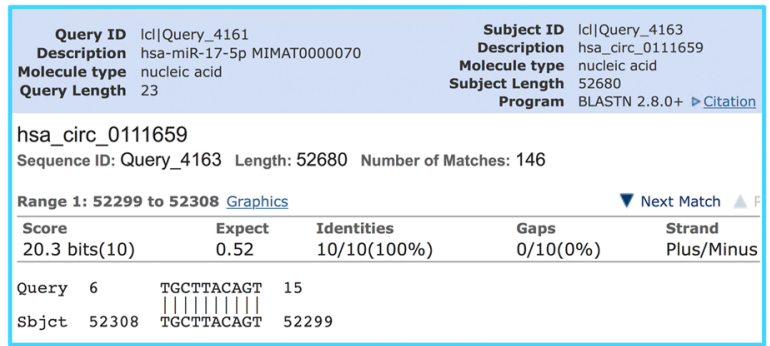

$\mathrm{b}_{3}$

\begin{tabular}{|c|c|c|c|c|}
\hline $\begin{aligned} & \text { Query ID }|c| \\
& \text { Description } \text { hs } \\
& \text { Molecule type nu } \\
& \text { Query Length } 23\end{aligned}$ & $\begin{array}{l}\text { c||Query_32583 } \\
\text { רsa-miR-93-5p MIMATOC } \\
\text { hucleic acid } \\
23\end{array}$ & & $\begin{array}{r}\text { Subject ID } \\
\text { Description } \\
\text { Molecule type } \\
\text { Subject Length } \\
\text { Program }\end{array}$ & $\begin{array}{l}\text { Ic|l|Query_4163 } \\
\text { hsa_cic_-0111659 } \\
\text { nucleic acid } \\
\text { 52680 } \\
\text { BLASTN 2.8.0+ DCitation }\end{array}$ \\
\hline $\begin{array}{l}\text { hsa_circ_011 } \\
\text { Sequence ID: Q }\end{array}$ & $\begin{array}{l}11659 \\
\text { 2uery_32585 Len }\end{array}$ & $: 52680$ Number & ches: 73 & \\
\hline \multicolumn{4}{|c|}{ Range 1: 9778 to 9788 Graphics } & V Next Match $\triangle$ \\
\hline $\begin{array}{l}\text { Score } \\
22.3 \text { bits(11) }\end{array}$ & $\begin{array}{l}\text { Expect } \\
0.13\end{array}$ & $\begin{array}{l}\text { Identities } \\
11 / 11(100 \%)\end{array}$ & $\begin{array}{l}\text { Gaps } \\
0 / 11(0 \%)\end{array}$ & $\begin{array}{l}\text { Strand } \\
\text { Plus/Minus }\end{array}$ \\
\hline Query 1 & CAAAGTGCTGT & & & \\
\hline Sbjet 9788 & CAAAGTGCTGT & 778 & & \\
\hline
\end{tabular}

Fig. 7 Prediction of circRNA-miRNAs-VEGF. (a) Molecular interactions between the VEGF gene and its target miRNAs. (b1-b3) Molecular interactions between hsa_circRNA_0111659 and its dominant target miRNAs. The common and dominant target miRNAs of hsa_circRNA_0111659 were miR-17-5p, miR-20b-5p, and miR-93-5p. CircRNAs: circular RNAs

proliferation. Interestingly, we also found that the levels of seven circRNAs in WJ-MSCs were lower than those in ESCs. This may be because ESCs can also secrete a small proportion of circRNAs, in addition to the circRNAs secreted by WJ-MSCs.

The function of circRNAs, which are transformed from their linear host RNAs, may be related to the known functions of their host linear transcripts. In our study, both the most significant biological processes, such as cell metabolic process, developmental process and biological regulation determined by GO analysis, and the most significant pathways such as DNA replication, cell cycle, homologous recombination, nucleotide excision repair, and AMPK signaling pathway determined by KEGG pathway analysis, were related to cell proliferation or DNA repair. Moreover, the host genes of the seven validated circRNAs were ASPM, MKI67, TROAP, WDR62, KIF14, and MYBL2. All of these genes are closely related to cell proliferation, the cell cycle, cell survival, and cell differentiation [27-33]. For example, one of the most upregulated circRNAs (hsa_circRNA_0111659) is derived from the KIF14 gene encoding a member of the kinesin family of proteins, which is involved in cell division, microtubule polymer dynamics, intracellular transportation, and signal transduction [32]. The developmental gene MKI67 produced three different circRNAs and encoded a protein that binds the perichromosomal layer during mitosis [28]. Higher levels of MKI67 expression in tumor tissue have been associated with a higher tumor grade and an earlier recurrence of disease [29]. We also found that the expression of seven circRNAs was not completely consistent with their host genes. A previous report has stated that most circular RNAs are rarely generated and accumulate in low levels [34]; however, some are expressed at levels tenfold higher than their associated linear mRNA [35]. Collectively, these results suggested that the main function of some protein-coding genes may be to generate circular RNAs rather than linear mRNAs or proteins. This conclusion would be logical because circRNAs, once produced, are naturally resistant to degradation by exonucleases and thus have long half-lives; moreover, some accumulate to high levels [36]. These upregulated circRNAs are derived from cell developmental genes involved in cell proliferation and growth factor signaling and may have other functions unrelated to their host genes such as binding specific miRNAs and regulating the expression of mRNA, or binding to other proteins and regulating their activities or functions [37-39]. Furthermore, in some cases, circRNAs can regulate the expression of their own host genes [40, 41]. However, it remains unclear whether this is a general mechanism among circRNAs.

From the circRNA-miRNA co-expression network, we found most of the circRNAs in the co-expression network had not been annotated as yet. It would therefore be very useful to perform further studies to reveal the underlying mechanisms of these circRNAs. CircRNAs might act as competing endogenous RNAs, thus regulating the function 
of miRNAs [19]. In our study, we predicted the differentially expressed circRNAs and found hsa_circRNA_0111659 possesses miR-17-5p, miR-20b-5p, and miR-93-5p binding sites, thus suggesting that hsa_circRNA_0111659 may be involved in the functional regulation of these three miRNAs in the process of repair by WJ-MSCs. Previous studies using PCR have also shown that the expression of miR-17-5p, miR-20b-5p, and miR-93-5p is downregulated in various types of cancer, thus suggesting that these act as tumor suppressors [42-50]. MiR-17-5p, one of the angiogenesis-associated miRNAs in human placentas, has been reported to be downregulated in endometriotic tissue $[51,52]$. These studies collectively indicate that the downregulated expression of miR-17-5p, miR-20b-5p, and miR-93-5p is associated with cell proliferation and tissue repair. The reduction in the expression of these miRNAs eases the post-transcriptional suppression of its target mRNA encoding the growth factor VEGF, thus promoting angiogenesis during the repair of endometrial lesions [53]. It has been suggested that the differentially expressed circRNAs, hsa_circRNA_0111659, act as miRNA sponges and may competitively bind miR-17-5p, miR-20b-5p, and miR-93-5p and release the target VEGF mRNA. However, the targeted verification of hsa_circRNA_0111659/miR-17-5p, miR-20b-5p, and miR-93-5p/ VEGF, as well as the function and molecular mechanism of hsa_circRNA_0111659 in the repair of endometrium by WJ-MSC, remains unclear and requires further investigation.

\section{Conclusions}

The present study is the first to identify the expression profile of circRNAs in the WJ-MSC-mediated repair of endometrial damage. We described circRNA expression patterns during the repair of endometrium damage by WJ-MSCs and found that more than 7000 circRNAs are differentially expressed in this process. Many circRNAs may participate in the biological pathways related to the repair of endometrial damage by different regulatory mechanisms. Moreover, we focused on hsa_circRNA_ 0111659 and predicted its miRNAs and targeted mRNA. The association of circRNA-miRNA-mRNA is likely to be involved in regulating the repair of endometrial damage. Our findings present a clearer understanding of the expression profile during WJ-MSC-mediated repair of the endometrium and provide a novel perspective for using WJ-MSCs effectively in the treatment of endometrial damage.

\section{Additional files}

Additional file 1: Table S1. Upregulated circRNAs in damaged ESCS from the cocultured group. Table S2. Downregulated circRNAs in damaged ESCs from the cocultured group. Table S3. GO BP analysis between cocultured group and non-cocultured group. Table S4. GO CC analysis between cocultured group and non-cocultured group. Table S5.
GO MF analysis between cocultured group and non-cocultured group. Table S6. Top 10 KEGG pathway analysis between cocultured group and non-cocultured group. Table S7. Top 10 circRNA_miRNAs. Table S8. Predicted miRNAs of VEGF by miRwalk database. (DOCX $1130 \mathrm{~kb}$ )

\section{Abbreviations}

BP: Biological process; CC: Cellular component; CircRNA: Circular RNA; ESC: Endometrial stromal cell; FBS: Fetal bovine serum; GO: Gene Ontology; KEGG: Kyoto Encyclopedia of Genes and Genomes; MF: Molecular function; miRNA: MicroRNA; NcRNA: Non-coding RNA; QPCR: Quantitative polymerase chain reaction; VEGF: Vascular endothelial growth factor; WJ-MSC: Wharton's jelly-derived mesenchymal stem cell

\section{Acknowledgements}

The authors would like to thank Dr. Qing Li and Yulong Liu of Capitalbio Technology, Beijing, China, for their precious help in analyzing the data of circRNA microarray.

\section{Funding}

This work was financially supported by the National Natural Science Foundation of China (No. 81771527), Social Development Foundation of Jiangsu Province, China (No. BE2018672), and Postdoctoral Science Foundation of Jiangsu Province (No. 2018K046A).

\section{Availability of data and materials}

All data generated or analyzed during this study are included in this paper.

Authors' contributions

BLS and YQZ conceived and designed the experiments. BLS, LS, QS, YJ, and ZYS performed the experiments. BLS, XQY, and YQZ analyzed the data. BLS, LS, and YQZ wrote the paper. LS and QS assisted in designing the study. All authors read and approved the final manuscript.

\section{Ethics approval and consent to participate}

This study was approved by the Institutional Review Board of the Affiliated Hospital of Nantong University. Written informed consent for the use of the tissue was obtained from each woman.

Consent for publication

Not applicable.

\section{Competing interests}

The authors declare that they have no competing interests.

\section{Publisher's Note}

Springer Nature remains neutral with regard to jurisdictional claims in published maps and institutional affiliations.

\section{Author details}

'Department of Obstetrics and Gynecology, Affiliated Hospital of Nantong University, Nantong, People's Republic of China. ${ }^{2}$ Department of Obstetrics and Gynecology, The Second Affiliated Hospital of Soochow University, Soochow, People's Republic of China. ${ }^{3}$ Department of Obstetrics and Gynecology, Affiliated Hospital of Nantong University School of Medicine, 19 Xishi Road, Nantong 226006, Jiangsu, People's Republic of China.

Received: 4 September 2018 Revised: 10 October 2018 Accepted: 14 October 2018 Published online: 15 November 2018

References

1. Herrler A, von Rango U, Beier HM. Embryo-maternal signalling: how the embryo starts talking to its mother to accomplish implantation. Reprod BioMed Online. 2003;6:244-56.

2. Norwitz ER. Defective implantation and placentation: laying the blueprint for pregnancy complications. Reprod BioMed Online. 2006;13:591-9.

3. Yen CF, Kim SH, Liao SK, Atabekoglu C, Uckac S, Arici A, et al. Increased expression of integrin-linked kinase during decidualization regulates the morphological transformation of endometrial stromal cells. Fertil Steril. 2017; 107:803-12. 
4. Evans J, Salamonsen LA, Winship A, Menkhorst E, Nie G, Gargett CE, et al. Fertile ground: human endometrial programming and lessons in health and disease. Nat Rev Endocrinel. 2016;12:654-67.

5. Singh N, Mohanty S, Seth T, Shankar M, Bhaskaran S, Dharmendra S. Autologous stem cell transplantation in refractory Asherman's syndrome: a novel cell based therapy. J Hum Reprod Sci. 2014;7:93-8.

6. Subramanian A, Fong CY, Biswas A, Bongso A. Comparative characterization of cells from the various compartments of the human umbilical cord shows that the Wharton's jelly compartment provides the best source of clinically utilizable mesenchymal stem cells. PLoS One. 2015;10:e0127992.

7. Rafieemehr H, Kheyrandish M, Soleimani M. Neuroprotective effects of transplanted mesenchymal stromal cells-derived human umbilical cord blood neural progenitor cells in EAE. Iran J Allergy Asthma Immunol. 2015; $14: 596-604$

8. Li X, Hu YD, Guo Y, Chen Y, Guo DX, Zhou HL, et al. Safety and efficacy of intracoronary human umbilical cord-derived mesenchymal stem cell treatment for very old patients with coronary chronic total occlusion. Curr Pharm Des. 2015;21:1426-32.

9. Hu J, Wang Y, Gong H, Yu C, Guo C, Wang F, et al. Long term effect and safety of Wharton's jelly-derived mesenchymal stem cells on type 2 diabetes. Exp Ther Med. 2016:12:1857-66.

10. Hendijan F, Javanmard SH, Sadeghi-aliabadi H. Human Wharton's jelly mesenchymal stem cell secretome display antiproliferative effect on leukemia cell line and produce additive cytotoxic effect in combination with doxorubicin. Tissue Cell. 2015;47:229-34.

11. Manzini BM, da Silva Santos Duarte A, Sankaramanivel S, Ramos AL, LatufFilho P, Escanhoela C, et al. Useful properties of undifferentiated mesenchymal stromal cells and adipose tissue as the source in liverregenerative therapy studied in an animal model of severe acute fulminant hepatitis. Cytotherapy. 2015;17:1052-65.

12. Pang $X$, Yang $H$, Peng B. Human umbilical cord mesenchymal stem cell transplantation for the treatment of chronic discogenic low back pain. Pain Physician. 2014;17:E525-30.

13. Yang $X$, Zhang M, Zhang Y, Li W, Yang B. Mesenchymal stem cells derived from Wharton jelly of the human umbilical cord amiliorate damage to human endometrial stromal cells. Fertil Steril. 2011;96:1029-36.

14. Chen X, Yang X, Wu R, Chen W, Xie H, Qian X, et al. Therapeutic effects of Wharton jelly-derived mesenchymal stem cells on a rat abortion models. J Obstet Gynaecol Res. 2016;42:972-82.

15. Memczak S, Jens M, Elefsinioti A, Torti F, Krueger J, Rybak A, et al. Circular RNAs are a large class of animal RNAs with regulatory potency. Nature. 2013:495:333-8.

16. Zhang $Y$, Zhang $X O$, Chen T, Xiang JF, Yin QF, Xing YH, et al. Circular intronic long noncoding RNAs. Mol Cell. 2013;51:792-806.

17. Li Z, Huang C, Bao C, Chen L, Lin M, Wang X, et al. Exon-intron circular RNAs regulate transcription in the nucleus. Nat Struct Mol Biol. 2015;22:256-64.

18. Rybak-Wolf A, Stottmeister C, Glazar P, Jens M, Pino N, Giusti S, et al. Circular RNAs in the mammalian brain are highly abundant, conserved, and dynamically expressed. Mol Cell. 2015;58:870-85.

19. Zheng Q, Bao C, Guo W, Li S, Chen J, Chen B, et al. Circular RNA profiling reveals an abundant circHIPK3 that regulates cell growth by sponging multiple miRNAs. Nat Commun. 2016;7:11215.

20. Hansen TB, Jensen TI, Clausen BH, Bramsen JB, Finsen B, Damgaard CK, et al. Natural RNA circles function as efficient microRNA sponges. Nature. 2013; 495:384-8.

21. Huo L, Zhang P, Li C, Rahim K, Hao X, Xiang B, et al. Genome-wide identification of circRNAs in pathogenic basidiomycetous yeast Cryptococcus neoformans suggests conserved circRNA host genes over kingdoms. Genes (Basel). 2018;9(3):118.

22. Li L, Guo J, Chen Y, Chang C, Xu C. Comprehensive circRNA expression profile and selection of key circRNAs during priming phase of rat liver regeneration. BMC Genomics. 2017;18:80.

23. Lin SP, Ye S, Long Y, Fan Y, Mao HF, Chen MT, et al. Circular RNA expression alterations are involved in OGD/R-induced neuron injury. Biochem Biophys Res Commun. 2016;471:52-6.

24. Du WW, Yang W, Liu E, Yang Z, Dhaliwai $P$, Yang BB. Foxo3 circular RNA retards cell cycle progression via forming ternary complexes with p21 and CDK2. Nucleic Acids Res. 2016:44:2846-58.

25. Caiment F, Gaj S, Claessen S, Kleinjans J. High-throughput data integration of RNA-miRNA-circRNAs reveals novel insights into mechanisms of benzo[a] pyrene-induced carcinogenicity. Nucleic Acids Res. 2015;43:2525-34.
26. Shi Q, Gao J, Jiang Y, Sun B, Lu W, Su M, et al. Differentiation of human umbilical cord Wharton's jelly derived masenchymal stems cell into endometrial cells. Stem Cell Res Ther. 2017;8:246.

27. Hagemann C, Anacker J, Gerngras S, Kühnel S, Said HM, Patel R, et al. Expression analysis of the autosomal recessive primary microcephaly genes MCPH1 (microcephalin) and MCPH5 (ASPM, abnormal spindle-like, microcephaly associated) in human malignant gliomas. Oncol Rep. 2008;20:301-8.

28. Naumova N, Imakaev M, Fudenberg G, Zhan Y, Lajoie BR, Mirny LA, et al. Organization of the mitotic chromosome. Science. 2013;342:948-53.

29. Nakanishi K, Sakamoto M, Yamasaki S, Todo S, Hirohashi S. Akt phosphorylation is a risk factor for early disease recurrence and poor prognosis in hepatocellular carcinoma. Cancer. 2005;103:307-12.

30. Yang S, Liu X, Yin Y, Fukuda MN, Zhou J. Tastin is required for bipolar spindle assembly and centrosome integrity during mitosis. FASEB J. 2008;22:1960-72.

31. Nicholas AK, Khurshid M, Désir J, Carvalho OP, Cox JJ, Thornton G, et al. WDR62 is associated with the spindle pole and is mutated in human microcephaly. Nat Genet. 2010:42:1010-4

32. Miki H, Okada Y, Hirokawa N. Analysis of the kinesin superfamily: insights into structure and function. Trends Cell Biol. 2005:15:467-76.

33. Musa J, Aynaud MM, Mirabeau O, Delattre O, Grünewald TG. MYBL2 (B-Myb): a central regulator of cell proliferation, cell survival and differentiation involved in tumorigenesis. Cell Death Dis. 2017;8:e2895.

34. Zhang XO, Dong $R$, Zhang $Y$, Zhang JL, Luo Z, Zhang J, et al. Diverse alternative back-splicing and alternative splicing landscape of circular RNAs. Genome Res. 2016;26:1277-87.

35. Jeck WR, Sorrentino JA, Wang K, Slevin MK, Burd CE, Liu J, et al. Circular RNAs are abundant, conserved, and associated with ALU repeats. RNA. 2013; 19:141-57.

36. Wilusz JE. A $360^{\circ}$ view of circular RNAs: from biogenesis to functions. Wiley interdiscip Rev RNA. 2018;9:e1478.

37. Ashwal-Fluss R, Meyer M, Pamudurti NR, Ivanov A, Bartok O, Hanan M, et al. circRNA biogenesis competes with pre-mRNA splicing. Mol Cell. 2014;56:55-66.

38. Chen YG, Satpathy AT, Chang HY. Gene regulation in the immune system by long noncoding RNAs. Nat Immunol. 2017;18:962-72.

39. Schneider T, Hung LH, Schreiner S, Starke S, Eckhof H, Rossbach O, et al. CircRNA-protein complexes: IMP3 protein component defines subfamily of circRNPs. Sci Rep. 2016;6:31313.

40. Abdelmohsen K, Panda AC, Munk R, Grammatikakis I, Dudekula DB, De S, et al. Identification of HuR target circular RNAs uncovers suppression of PABPN1 translation by CircPABPN1. RNA Biol. 2017;14:361-9.

41. Heumüller AW, Boeckel JN. Characerization and validation of circular RNA and their host gene mRNA expression using PCR. Methods Mol Biol. 2018; 1724:57-67.

42. Sueta A, Yamamoto Y, Tomiguchi M, Takeshita T, Yamamoto-lbusuki M, Iwase H. Differential expression of exosomal miRNAs between breast cancer patients with and without recurrence. Oncotarget. 2017;8:69934-44.

43. Chen $X$, Shi $K$, Wang Y, Song M, Zhou W, Tu H, et al. Clinical value of integrated-signature miRNAs in colorectal cancer: miRNA expression profiling analysis and experimental validation. Oncotarget. 2015;6:37544-56.

44. McDermott AM, Miller N, Wall D, Martyn LM, Ball G, Sweeney KJ, et al. Identification and validation of oncologic miRNA biomarkers for luminal A-like breast cancer. PLoS One. 2014;9:e87032.

45. Hu J, Xu J, Wu Y, Chen Q, Zheng W, Lu X, et al. Identification of microRNA-93 as a functional dysregulated miRNA in triple-negative breast cancer. Tumour Biol. 2015;36:251-8.

46. Guo J, Meng R, Yin Z, Li P, Zhou R, Zhang S, et al. A serum microRNA signature as a prognostic factor for patients with advanced NSCLC and its association with tissue microRNA expression profiles. Mol Med Rep. 2016;13: 4643-53.

47. Wang J, Tian X, Han R, Zhang X, Wang X, Shen H, et al. Downregulation of miR-486-5p contributes to tumor progression and metastasis by targeting protumorigenic ARHGAP5 in lung cancer. Oncogene. 2014;33:1181-9.

48. Zhang Y, Zhang D, Wang F, Xu D, Guo Y, Cui W. Serum miRNAs panel (miR16-2*, miR-195, miR-2861, miR-497) as novel non-invasive biomarkers for detection of cervical cancer. Sci Rep. 2015;5:17942.

49. Liu L, Ye JX, Qin YZ, Chen QH, Ge LY. Evaluation of miR-29c, miR-124, miR-135a and miR-148a in predicting lymph node metastasis and tumor stage of gastric cancer. Int J Clin Exp Med. 2015;8:22227-36.

50. Fiore D, Donnarumma E, Roscigno G, laboni M, Russo V, Affinito A, et al. miR-340 predicts glioblastoma survival and modulates key cancer hallmarks through down-regulation of NRAS. Oncotarget. 2016;7:19531-47. 
51. Jia SZ, Yang Y, Lang J, Sun P, Leng J. Plasma miR-17-5p, miR-20a and miR-22 are down-regulated in women with endometriosis. Hum Reprod. 2013;28:322-30.

52. Ramón LA, Braza-Boïls A, Gilabert-Estellés J, Gilabert J, España F, Chirivella M, et al. MicroRNAs expression in endometriosis and their relation to angiogenic factors. Hum Reprod. 2011;6:1082-90.

53. Paiva P, Hannan NJ, Hincks C, Meehan KL, Pruysers E, Dimitriadis E, et al. Human chorionic gonadotrophin regulates FGF2 and other cytokines produced by human endometrial epithelial cells, providing a mechanism for enhancing endometrial receptivity. Hum Reprod. 2011;26:1153-62.

Ready to submit your research? Choose BMC and benefit from:

- fast, convenient online submission

- thorough peer review by experienced researchers in your field

- rapid publication on acceptance

- support for research data, including large and complex data types

- gold Open Access which fosters wider collaboration and increased citations

- maximum visibility for your research: over $100 \mathrm{M}$ website views per year

At BMC, research is always in progress.

Learn more biomedcentral.com/submissions 